\title{
Potential National Security Applications of Nuclear Resonance Fluorescence
}

\author{
GA Warren \\ PN Peplowski \\ JA Caggiano
}

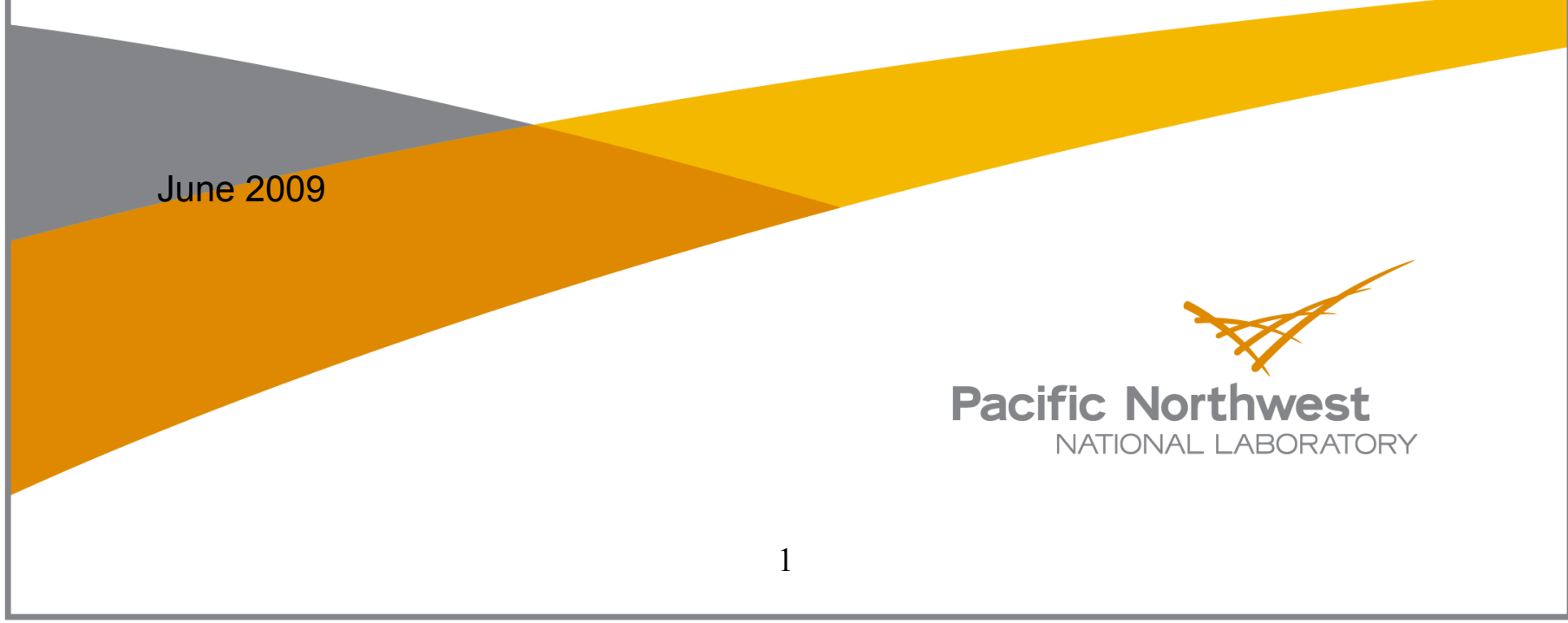




\title{
DISCLAIMER
}

This report was prepared as an account of work sponsored by an agency of the United States Government. Neither the United States Government nor any agency thereof, nor Battelle Memorial Institute, nor any of their employees, makes any warranty, express or implied, or assumes any legal liability or responsibility for the accuracy, completeness, or usefulness of any information, apparatus, product, or process disclosed, or represents that its use would not infringe privately owned rights. Reference herein to any specific commercial product, process, or service by trade name, trademark, manufacturer, or otherwise does not necessarily constitute or imply its endorsement, recommendation, or favoring by the United States Government or any agency thereof, or Battelle Memorial Institute. The views and opinions of authors expressed herein do not necessarily state or reflect those of the United States Government or any agency thereof.

\author{
PACIFIC NORTHWEST NATIONAL LABORATORY \\ operated by \\ BATTELLE \\ for the \\ UNITED STATES DEPARTMENT OF ENERGY \\ under Contract DE-AC05-76RL01830
}

Printed in the United States of America
Available to DOE and DOE contractors from the Office of Scientific and Technical Information,
P.O. Box 62, Oak Ridge, TN 37831-0062;
ph: (865) 576-8401
fax: $(865)$ 576-5728
email: reports@adonis.osti.gov

\begin{abstract}
Available to the public from the National Technical Information Service, U.S. Department of Commerce, 5285 Port Royal Rd., Springfield, VA 22161 ph: (800) 553-6847 fax: $(703) 605-6900$ email: orders@ntis.fedworld.gov online ordering: http://www.ntis.gov/ordering.htm
\end{abstract}

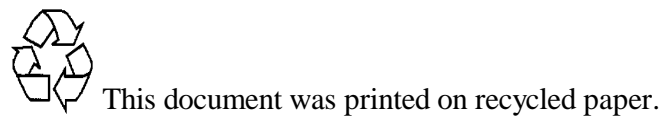




\section{Potential National Security Applications of Nuclear Resonance Fluorescence}

GA Warren

PN Peplowski

JA Caggiano

June 2009 


\section{Executive Summary}

Nuclear Resonance Fluorescence (NRF) is a physics process that has potential to address numerous and varied national security applications. With the use of an incident photon beam, NRF provides isotope-specific signatures for a wide variety of materials that can be used to detect and characterize samples. Exploitation of these signatures may lead to new applications.

The objective of this report is to document the initial investigation into the possible research issues related to the development of NRF-based national security applications. These potential applications include

- Measuring uranium enrichment in UF6 canisters

- Geo-sourcing of material

- Weapons dismantlement verification

- Direct measurement of Pu in spent fuel

- Self-irradiating samples

- Gas samples

- Tagging

- Characterization of suspect material

- Verification of special nuclear material in a cargo container

While these applications are varied, there are only a few research issues that need to be addressed to understand the limitation of NRF in solving these problems. These research issues include

- Source intensity

- Detector resolution

- Detector rate

- Measuring small samples

- Measuring small quantities of isotopes within a larger matrix

- Three-dimensional isotopic mapping

- Sensitivity through layers of materials

- Measuring through large quantities of materials

The next effort will determine how best to answer the research issues, followed by a prioritization of those questions to ensure that the most important are addressed. These issues will be addressed through either analytical calculations, computer simulations, analysis of previous data or collection of new measurements. It will also be beneficial to conduct a thorough examination of a couple of the more promising applications in order to develop concrete examples of how NRF may be applied in specific situations. The goals are to understand whether the application of NRF is limited by technology or physics, to conduct a limited exploration of approaches to reduce the technical limitations, and to develop a research roadmap to provide guidance for the direction of future NRF research so that NRF may be used to address national security issues. 


\section{Acronyms}

CT Computed tomography

CW Continuous-wave

HE High Explosives

HEU Highly-enriched uranium

HIGS High Intensity Gamma Source

HPGe High-purity germanium

IAEA International Atomic Energy Agency

LLNL Lawrence Livermore National Laboratory

NRF Nuclear Resonance Fluorescence

PNNL Pacific Northwest National Laboratory

SNM Special nuclear materials 


\section{Contents}

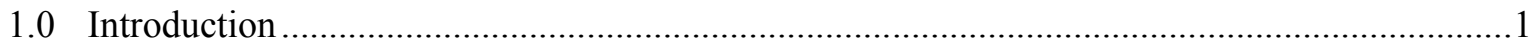

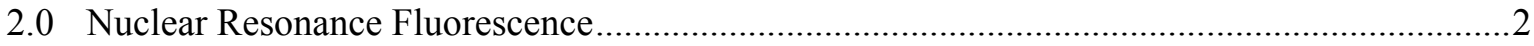

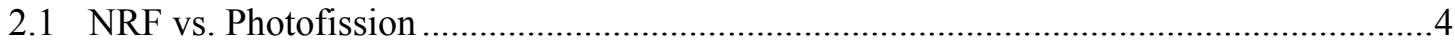

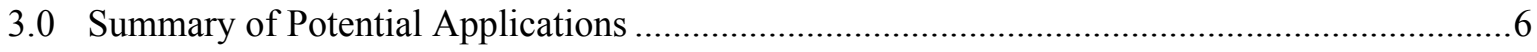

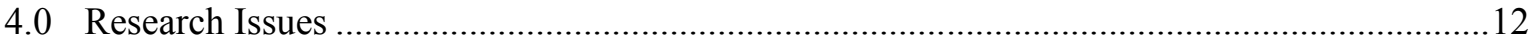

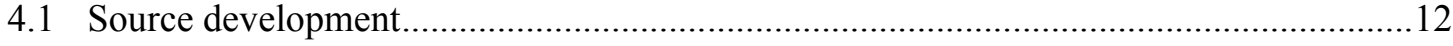

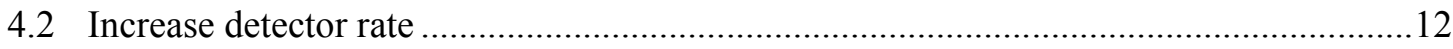

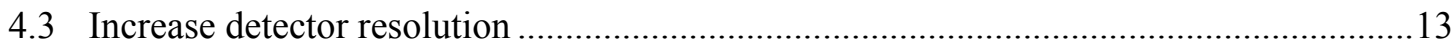

4.4 Sensitivity to small quantities of material …...............................................................

4.5 Sensitivity to small quantities of isotopes within larger matrix ....................................13

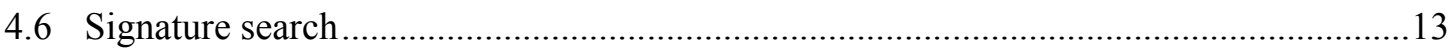

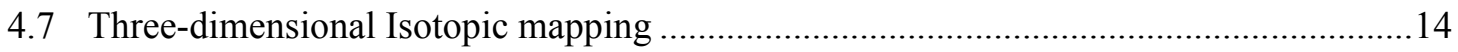

4.8 Sensitivity through layers of materials ..................................................................... 14

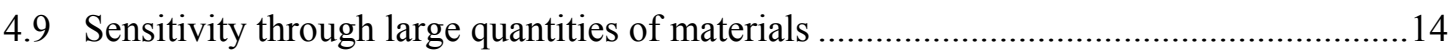

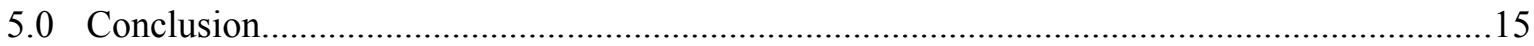

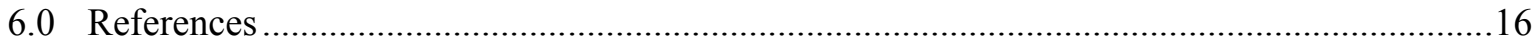




\subsection{Introduction}

Nuclear Resonance Fluorescence (NRF) is a physics process that has potential to address numerous and varied national security applications. With the use of an incident photon beam, NRF provides isotope-specific signatures for a wide variety of materials that can be used to detect and characterize samples. Exploitation of these signatures may lead to new applications.

NRF has been used in the physics lab as a tool to study nuclear structure for more than five decades. The seminal paper describing the physics and measurement techniques is Metzger [1959]. More recently, discovery of the scissor states ${ }^{1}$ of nuclei was conducted using NRF [Boehle, 1984]. Advances in accelerator technology, enabling high-flux, high duty-factor electron beams, and in detector technology, enabling high-resolution gamma-ray detection, permit the extension of this laboratory tool to practical applications. The first proposal of the use of NRF as a tool to detect special nuclear materials within cargo containers is generally credited to Prof. William Bertozzi at the Massachusetts Institute of Technology [Bertozzi, 2005]. Over the last few years, several organizations, including Passport Systems, Inc., Pacific Northwest National Laboratory (PNNL) and Lawrence Livermore National Laboratory (LLNL) have explored various research issues related to the application of NRF. For instance, Passport, PNNL and LLNL collaborated on the discovery of the first NRF signatures of ${ }^{235} \mathrm{U}$ and ${ }^{239} \mathrm{Pu}$ [Bertozzi, 2008].

The objective of this report is to document the initial investigation into the possible research issues related to the development of NRF-based national security applications. In the initial phase of this project, potential applications were gathered from the NA-22 Special Nuclear Material Movement Detection Portfolio Roadmap [Roadmap, 2007] and interviews with PNNL staff familiar with the various research needs related to national security. While these applications are varied, there are only a few overlapping research issues that need to be addressed in order to develop a better understanding of the capabilities and limitations of NRF. By investigating these issues, one has the potential to advance the understanding of NRF applications across a broad assortment of applications.

This report is divided into four sections. Following this introduction, a general description of the NRF physics process is provided. Included in this description is a brief comparison of NRF to photofission. The next section provides a brief description of the varied potential applications that could be addressed by NRF. The final section of this report discusses those common research issues.

\footnotetext{
${ }^{1}$ In the scissor states, the excited nucleus vibrates like a pair of scissors opening and closing in which one arm is composed of neutrons and the other of protons.
} 


\subsection{Nuclear Resonance Fluorescence}

$\mathrm{NRF}$ is an active interrogation technique that can provide isotope-specific information. In the interrogation process, illustrated in Fig. 1, a sample is illuminated with a photon beam. The photons are typically in the 1.5 to $8 \mathrm{MeV}$ energy range. The nuclei of the sample will absorb photons at very specific energies, leaving the nuclei in an excited level. That energy level then decays, following normal cascade paths, back down to the ground state. The emitted gamma rays can then be observed and used to detect and/or characterize the isotopic content of the sample.

NRF has a few critical properties that make it very attractive as an active interrogation technique. These properties are described below:

- The observed gamma rays are characteristic of specific isotopes.

- The process is not confined to special nuclear materials (SNM). In fact, many isotopes have observable NRF signatures.

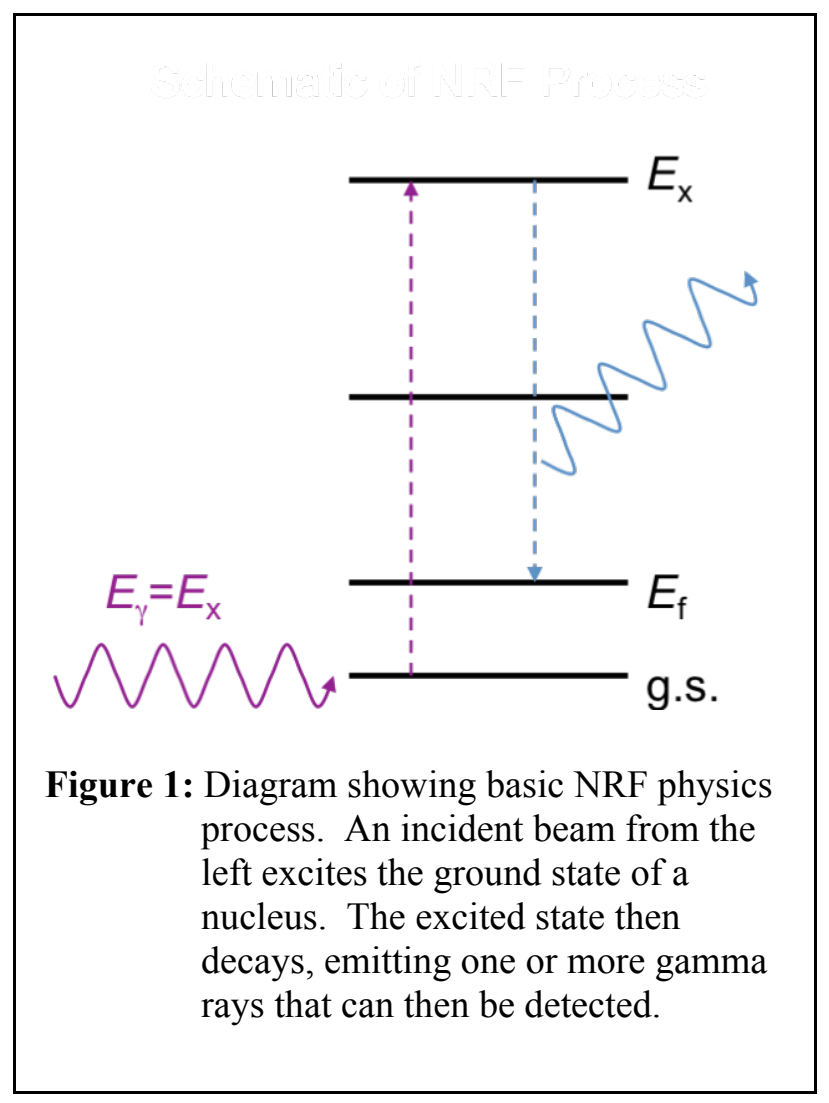

- The energies of the gamma-ray signatures are in the range of a few $\mathrm{MeV}$, which enables the penetration of significant amounts of material. For high Z materials, NRF signatures fall near the minima of the atomic cross sections.

- The process is very strong. The NRF cross sections are comparable to or even larger than atomic cross sections in many cases. At their maximum, the NRF cross sections are also roughly one to two orders of magnitude stronger than photofission cross sections.

- The resonances are very narrow. After Doppler broadening, the resonances are typically an $\sim \mathrm{eV}$ wide.

The two primary devices of an NRF measurement are the photon source and the gamma-ray detector. Traditionally, the photon source has been a high energy bremsstrahlung source. For this source, electrons in the $\mathrm{MeV}$ energy range are directed onto a metal plate. As the electrons slow down in the plate material, a continuous distribution of photons with energies up to the electron energy is generated. The disadvantage of this type of source is that a significant amount of the photon flux cannot be used to generate a NRF signal. This extra flux increases both dose to the sample and background in the measurement.

A second class of source generates photons with a narrow range of energies. There are several techniques to accomplish this approach, but one of the more common techniques is Compton backscattering. For these sources, photons, typically from a laser, are deflected 180 degrees by a high energy electron beam, typically hundreds of $\mathrm{MeV}$ in energy, to generate $\mathrm{MeV}$-scale photons. While this source provides a significantly higher fraction of incident photons within the resonance than a bremsstrahlung source, it also is a significantly more complex instrument. An 
example of a narrow bandwidth source is the High Intensity Gamma Source (HIGS) at Duke University.

The gamma-ray detector measures the photons generated from the photon beam incident on the target. These "scattered" photons are generated from both the NRF process and from typical electronic processes in which the incident photon interacts with the atomic electrons of the sample. Given the narrow energy width of the resonances, the typical NRF measurement setup deploys high purity germanium (HPGe) detectors. These detectors provide both high resolution and high intrinsic efficiency for the desired $\mathrm{MeV}$-range photons. Unfortunately, the relatively slow response of these detectors, on the scale of microseconds, limits the instantaneous rate to which the detector can be exposed. It is conceivable that for certain special applications, the high resolution of HPGe detectors may not be required. In such cases, other photon detectors that are less expensive and more operationally robust may provide adequate performance.

The rate limitation of HPGe detectors restricts the type of photon sources. For NRF measurements, determining the energy of the photon is critical. If the scattered photon rate is too high, then there is pileup in the detector. Pileup affects the measurement by both removing events from the peak and increasing the background under the peak, significantly degrading the quality of the measurement. To reduce the problems of pileup, one usually uses a photon source that is continuous on the time scale of detector response time (time structure faster than a microsecond). For a bremsstrahlung source, one typically uses a continuous-wave (CW) electron beam. Unfortunately, this restriction limits the research facilities in which one can conduct these measurements, as the majority of electron accelerators are pulsed, with time structure on the scale of milliseconds.

For this review, both the bremsstrahlung photon source and a narrow bandwidth photon source will be considered. Each source has its advantages and disadvantages for NRF measurements. While design of a photon source is beyond the scope of this work, specifications of a photon source to address a specific application will be addressed in future efforts.

There are basic NRF measurement modes, as shown in Figure 2. For the scattering setup, the incident photon beam creates NRF gamma rays that are directly measured by the scattering detector. Generally, the NRF gamma rays are detected at backward angles with respect to the beam to reduce the background contributions. For transmission measurements, the incident photon beam passes through the sample and then through a secondary target containing the isotopes of interest. If those isotopes are in the sample, then the NRF process will reduce the photon flux of the transmitted photons on the resonant energy creating a narrow "notch" in the energy spectrum. When the transmitted photons then pass through the secondary target, there will be fewer photons on resonance to generate an NRF response from the secondary target, so that the NRF peak in the transmission detector will have fewer counts.

The objective of this project is to investigate potential NRF applications and determine the research path that is necessary to develop the technologies to enable those applications. As there are advantages and disadvantages to different types of detectors, different types of sources and different measurement modes, this effort will consider all of these possibilities to find the approach must suitable for a particular application. 


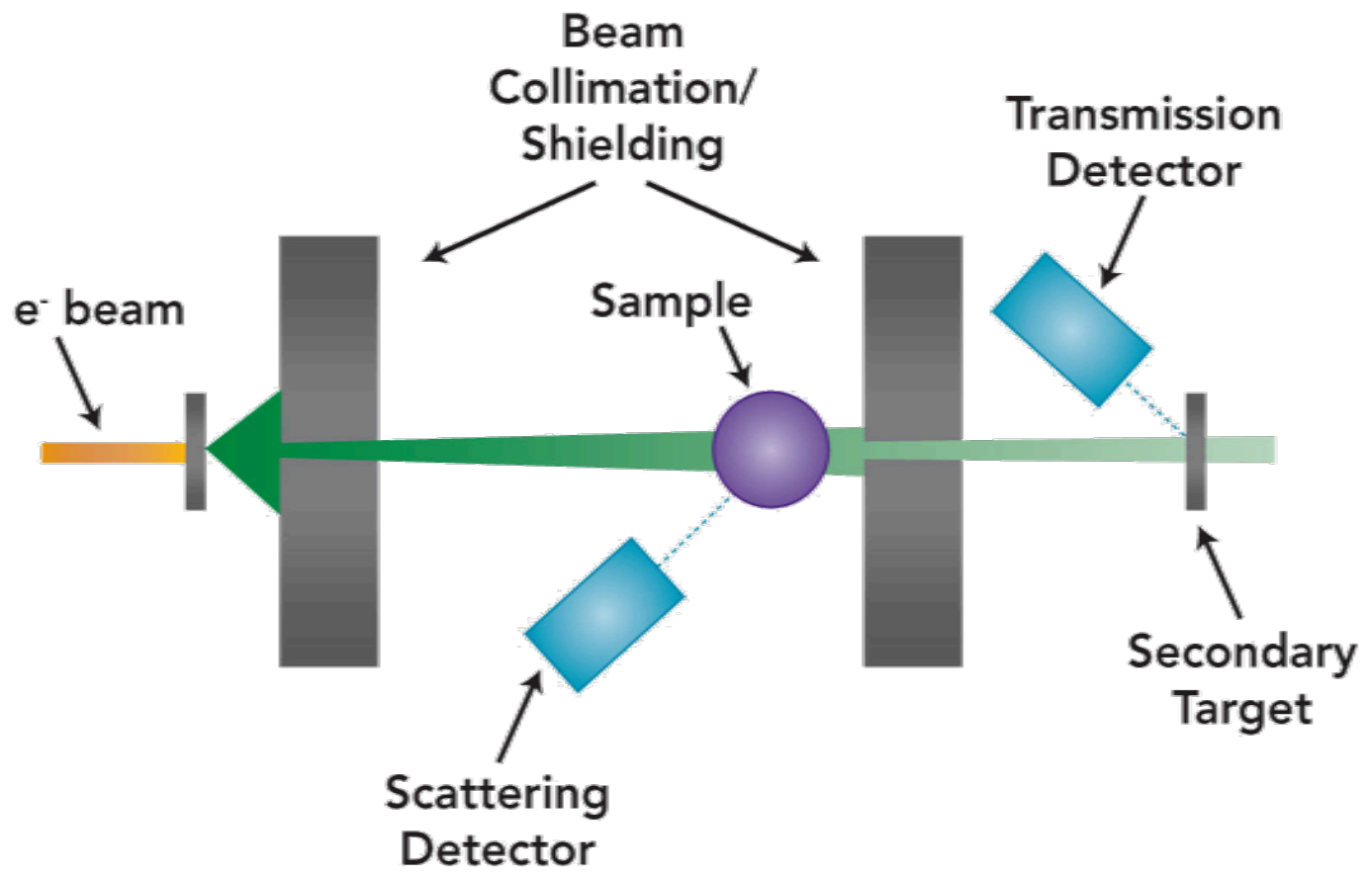

Figure 2: Schematic showing scattering and transmission NRF setups. The electron comes in from the left of the page, strikes the radiator to create a photon beam.

\subsection{NRF vs. Photofission}

The focus of the initial research on applying NRF techniques to national and homeland security applications was on detection of shielded SNM. Given this application, it is natural to compare NRF to photofission, which shares the same type of interrogating source as NRF. In photofission, photons above the photofission threshold induce fission when they are absorbed by fissionable nuclei. The fission creates a variety of observables that can be exploited for both detection and characterization applications.

The photofission observables can be classified by the time scale and particle. There are prompt particles emitted when the fission occurs, and there are also delayed particles generated by either fission products or additional fissions generated by the fission neutrons of the initial reaction. For practical applications, the two particles that will be considered are neutrons and photons; electrons and fission products are also produced, but they have an extremely limited range inside material so that they are difficult to observe.

For this paper, we will focus on the prompt neutrons, which provide the strongest and most selective signature generated by photofission. The prompt particles are a couple of orders of magnitude more numerous than the delayed particles [Snyder, 1951]. High-energy photons are generated by many different processes, from Compton scattering to pair-production as well as fission, so that they lack process-specificity. Neutrons, on the other hand can be created by two processes, photofission and neutron knockout $((\gamma, \mathrm{f})$ and $(\gamma, \mathrm{n}))$. Both of these are threshold processes that require the incident photon to exceed a certain value before the process can occur. It is generally the case that the $(\gamma, \mathrm{f})$ thresholds in SNM are lower than the $(\gamma, \mathrm{n})$ thresholds of most 
materials, with the notable exception of materials like ${ }^{2} \mathrm{H},{ }^{9} \mathrm{Be},{ }^{13} \mathrm{C},{ }^{17} \mathrm{O}$. This lower threshold for the photofission process provides limited process selectivity.

In contrast, NRF generates only one type of signal, namely prompt gamma rays at very specific energies depending on the isotope. The only way to lose this specificity is if two isotopes within the sample have very similar resonant energies. In this situation, the overlapping resonances can be resolved by considering other NRF lines of the isotopes. ${ }^{2}$ In such a situation, measurements can be conducted in a manner that would require overlapping on the scale of an $\mathrm{eV}$, which virtually eliminates any possibility of interference.

It is instructive to compare the raw signal-generation rate from these two processes. Consider an example in which a bremsstrahlung photon beam is incident on a $1 \mathrm{~cm}$ thick plate of ${ }^{235} \mathrm{U}$. We estimated the number of signature particles generated inside this plate of uranium. To keep this comparison as simple as possible, we ignored detector efficiency and solid angle. Assumptions made for the calculations at times favored the NRF process. Despite this tipping of the playing field, we concluded that for raw detection capability photofission provides significantly more signal.

The photon source was assumed to be a bremsstrahlung beam for both processes. The radiator was modeled as a $1-\mathrm{cm}$ thick $\mathrm{Cu}$ plate, which was followed by a $40-\mathrm{cm}$ thick lead collimator with a 2-cm diameter aperture. The end-point energy was $2.2 \mathrm{MeV}$ for the NRF process and $10 \mathrm{MeV}$ for the photofission process. The end-point energy for NRF was based on experimental experience for the optimum energy for measuring the NRF signature of ${ }^{235} \mathrm{U}$. The end-point energy for photofission was chosen to remain below the $(\gamma, n)$ threshold for most materials. This end-point energy is below the maximum cross section for photofission, which peaks near $15 \mathrm{MeV}$; one could achieve higher signal generation rates for photofission with a higher energy, but at the cost of specificity. We assumed constant beam power for the two bremsstrahlung sources, in effect increasing the electron beam current by a factor of 4.5 for the NRF calculation relative to the photofission calculation.

Attenuation of the incident beam in the uranium plate was taken into account. For NRF, the cross section will be in saturation, i.e. the thin-target approximation is not valid, so that incorporating the exponential loss of the incident photon flux is important. For photofission, the loss is less significant so that attenuation is incorporated by correcting for the absorption of the incident flux at the center of the uranium plate.

The NRF signal generation rate was calculated following Metzger [1959]. The calculation incorporates the effects of beam attenuation in the sample due to resonant and electronic processes as well as Doppler broadening of the resonance. The calculation was modified to ignore the attenuation of the fluorescent photons escaping the sample. The photon flux per $\mu \mathrm{C}$ of electron beam current was calculated using Geant 4 to model the bremsstrahlung source. It was determined that there would be $24 \mathrm{NRF}$ interactions per $\mu \mathrm{C}$ of electron beam incident on the bremsstrahlung radiator for the $1733-\mathrm{keV}$ resonance of ${ }^{235} \mathrm{U}$. There are several other resonances of ${ }^{235} \mathrm{U}$, so that with a $2.2-\mathrm{MeV}$ beam one would expect roughly 1 of these other photons for every $1733-\mathrm{keV}$ photon. Thus, incorporating the factor of 4.5 to maintain the same source power,

\footnotetext{
${ }^{2}$ The authors are aware of several examples of overlapping resonances, but they are unaware of any that cannot be resolved through comparison with other resonances. However, this does not mean that a few pathological cases cannot exist.
} 
one can expect roughly $220 \mathrm{NRF}$ photons to be generated per $\mu \mathrm{C}$ equivalent of a $10-\mathrm{MeV}$ bremsstrahlung source.

The photofission signal generation rate was calculated by considering the energy dependence of the photon flux, the photofission cross section, and the incident beam attenuation. The energy dependence of the photon flux was determined from simulations of a $10-\mathrm{MeV}$ end-point energy. The energy dependence of the evaluated photofission cross section was taken from Varlamov [1987]. The attenuation of the incident beam was determined using the total coherent electronic cross section of the photons in uranium from XCOM [2009]. The electronic cross section was far greater than either the $(\gamma, f)$ or $(\gamma, n)$ cross sections, so that only the electronic cross section was incorporated into the attenuation calculation. The choice of the total coherent cross section will overestimate the importance of the incident beam attenuation due to the inclusion of low-angle Compton scattering, which will have virtually no impact on photofission rate. The photofission rate for this setup was determined to be $5.7 \cdot 10^{5}$ interaction $/ \mu \mathrm{C}$ of electron beam. Given that approximately 2.4 neutrons are generated per fission [Vandendosch, 1973], this implies a signal generation rate of $1.4 \cdot 10^{6}$ per $\mu \mathrm{C}$ of electron beam. Thus, for this simple comparison one can expect a signal roughly 6000 times stronger from photofission than from NRF.

Signal strength is not the only consideration when one chooses which physics process to exploit when developing an application. While photofission may provide a stronger raw signal, the isotopic specificity of NRF may be valuable for specific applications. For instance, if one observes neutrons using a high-energy bremsstrahlung beam, it is difficult to differentiate whether the source was from photofission of fissionable materials or from $(\gamma, n)$ processes, or some combination of the two. In contrast, the NRF observables provide an isotope-specific answer. In addition, NRF is not limited to fissionable materials, as is photofission, but can be used to detect/characterize most common materials including lead and boron, two common materials used in shielding. Regardless of these fine details of specificity, we conclude that for applications requiring raw detection of fissionable material, without the need to identify which fissionable isotope nor the need to discriminate from possible background processes, photofission will provide a significantly stronger observable than NRF. A more thorough comparison between the two processes would need to be conducted in which false positives or fissionable material discrimination is important.

\subsection{Summary of Potential Applications}

Concepts for potential applications were gathered from a variety of sources. These included PNNL staff familiar with a range of national security needs, the NA-22 SNM movement Roadmap, and the authors' experience with NRF. The primary source was interviews with PNNL staff. During these discussions, the basic concept of NRF was introduced followed by a discussion of how the capabilities of NRF may address national security needs with which the particular staff member was familiar. During these discussions, the interviewees were encouraged to not limit concepts by perceived limitations of NRF. For instance, conducting NRF laboratory measurements on a 1-gram sample is currently a challenge; numerous applications were suggested that involve quantities of target material several orders of magnitude smaller. This initial lack-of-limitations approach was taken so that potential research issues could be more easily identified.

To help differentiate NRF from existing technologies, applications were screened to require nonintrusive evaluation and isotopic information. By non-intrusive, we imply sealed measurement samples or samples than one cannot directly access. For instance, if one could destroy a small 
portion of the sample, then it is quite likely that existing technologies, such as mass spectrometry, would outperform NRF techniques. Other potential applications were selected because NRF may provide a faster and/or less expensive method to determine the isotopic content.

A brief description of several of the more interesting applications follows. Each application includes a description of the problem, a scenario of how NRF may be used to address the problem, and finally a list of technical challenges. For each of these applications, it is assumed that signal rate and signal-to-noise are research issues that needs to be addressed.

\section{- U enrichment confirmation of $U F_{6}$ canisters}

Enriched uranium is frequently transported in canisters as $\mathrm{UF}_{6}$. The dimensions of the canisters can vary from fractions of a liter to thousands of liters. With the relatively low energy emissions of ${ }^{235} \mathrm{U}$, it is difficult to assay these containers to confirm that the stated uranium enrichment is correct. For instance, the $186-\mathrm{keV}$ gamma ray of ${ }^{235} \mathrm{U}$ will be attenuated by $95 \%$ in $6.5 \mathrm{~mm}$ of $\mathrm{UF}_{6}$ at a density of $3 \mathrm{~g} / \mathrm{cm}^{3}$. This range is insignificant compared to the $600 \mathrm{~mm}$ radius of the larger canisters. The $1001-\mathrm{keV}$ line of ${ }^{238} \mathrm{U}$ does provide for significantly more penetration through material than the ${ }^{235} \mathrm{U}$ signature. However, ${ }^{238} \mathrm{U}$ is only part of the determination of enrichment so that a direct measurement of ${ }^{235} \mathrm{U}$ is still desirable.

NRF has the potential to determine uranium enrichment inside a UF6 container for two reasons. The relevant energies using NRF allow for significantly more penetration through material. The attenuation length at $1733-\mathrm{keV}$, the strongest $\mathrm{NRF}$ line of ${ }^{235} \mathrm{U}$, is 30 times longer for the $186-\mathrm{keV}$ line. The second reason is that the NRF signal intensity can be increased by increasing the intensity of the photon source; an option not available when relying on a passive measurement.

The scenario for this application is an in-field standoff measurement to determine the enrichment of uranium in the canister. The canister will be roughly $3 \mathrm{~m}$ from both the source and the detector. The measurement will need to be completed on the time scale of an hour. Both type 5B and $48 \mathrm{Y}$ canisters would be examined.

The technical challenges are

- Sensitivity through large quantities of materials

- Sensitivity through multiple layers of materials

- Sensitivity to small quantities of isotopes in a larger matrix

- Geo-sourcing of material $(\mathrm{Cu}, \mathrm{Pb})$

There are many instances in which the geographic origin of a material can provide critical information for decision-makers. One approach to geo-sourcing a material is to look at ratios of isotopes. Moody [2005] shows a correlation between the ratio of ${ }^{207} \mathrm{~Pb}$ and ${ }^{206} \mathrm{~Pb}$ in aerosols and gasoline and the geographic location. Another example provided by Moody [2005] is the dependence in uranium oxide fuel of the ${ }^{18} \mathrm{O} /{ }^{16} \mathrm{O}$ ratio on geographic location where the $\mathrm{UF}_{6}$ was converted to uranium oxide.

The NRF process generates isotopic signatures for many of the isotopes of interest. For example, both ${ }^{206} \mathrm{~Pb}$ and ${ }^{207} \mathrm{~Pb}$ have a response around $4 \mathrm{MeV}$, while the ${ }^{16} \mathrm{O}$ response is around $7 \mathrm{MeV}$ and the ${ }^{18} \mathrm{O}$ is around $6 \mathrm{MeV}$. 
The scenario envisioned for this application is the laboratory measurements of lead and copper. We consider two cases, that of a lead sheet and a copper pipe. The measurement will need to be completed on the time scale of one day. The research issue is whether NRF can provide an answer more quickly and easily than existing techniques that may require significant laboratory work to complete.

The technical challenges are

- Sensitivity to small quantities of isotopes in a larger matrix

\section{- Weapons dismantlement verification}

There are two critical stages in the weapons dismantlement process at which NRF may provide invaluable verification capabilities. First, there is verification that the assembled weapon is actually a weapon. In principle, the NRF process can be exploited to confirm several attributes of SNM such as presence, form, geometry, isotopic content and fissile mass as well as attributes of high explosives (HE) such as presence, geometry and separation from fissile material. The second stage at which NRF could prove invaluable is the confirmation that the components of the disassembled weapon are consistent with the weapon. The disassembled components are stored in sealed containers so that direct access is not possible. However, NRF techniques could penetrate the container walls.

Another advantage of NRF is the potential for selectivity for individual isotopes. For transmission measurements, the secondary target must contain the isotopes of interest. By selecting suitable samples, one can limit the isotopic information available from these types of measurements. This capability may be critical as the isotopic content of weapons material is considered classified by some foreign governments.

The scenario for this application is a dedicated, fixed system installed at a facility. Measurements need to be conducted on the time scale of hours, and the geometry can be as close as allowed by the sample geometries.

The technical challenges are

- Sensitivity through multiple layers of materials

- Sensitivity to small quantities of isotopes in larger matrix

- Sensitivity through large quantities of materials

- Three-dimensional isotopic mapping

- Lack of knowledge of NRF signatures of some isotopes, namely plutonium isotopes other than ${ }^{239} \mathrm{Pu}$ and ${ }^{6} \mathrm{Li}$ and ${ }^{9} \mathrm{Be}$

\section{- Direct measurement of Pu in spent fuel}

One significant issue in safeguarding nuclear fuel is the proper accounting for the $\mathrm{Pu}$ content in the fuel. Current approaches rely on measurements of long-lived and non-fissile isotopes (e.g., ${ }^{137} \mathrm{Cs}$ ) coupled to burn-up code calculations to infer the isotopic content of the fuel. The measurements provide a confirmation of the operator-provided information. This approach falls well short of the direct and operator-independent measurements of $\mathrm{Pu}$ in spent fuel requested by the International Atomic Energy Agency (IAEA) [Smith, 2008].

One of the challenges of conducting measurements on spent fuel is its high activity, which can easily overwhelm a HPGe detector if proper collimation and shielding are not addressed. 
It has been shown that through careful selection of beam energy it may be possible to generate NRF signatures that can be observed within the background of the spent fuel [Quitter, 2008] .

The scenario envisioned for this application is the inspection of a Westinghouse $17 \times 17$ pin assembly. The fuel is an oxide with a density of $11 \mathrm{~g} / \mathrm{cm}^{3}$. The nominal target measurement uncertainty is $2 \%$ without any significant time constraints on the measurements (e.g. on the order of one day to measure). The detector is assumed to be $1 \mathrm{~m}$ away.

The technical challenges are

- High background rate from spent fuel

- Sensitivity through large quantities of materials

- Lack of knowledge of NRF signatures of isotopes

- Self-Irradiating Samples

Consider a sample that is very radioactive with a wide variety of isotopes so that many different energy gamma rays are emitted. If the sample is either large enough or is in a matrix of material, then there will be significant downscattering of those gamma rays which will produce a quasi-continuous distribution of photons. In this case, those photons could induce NRF within the sample.

This geometry is significant different from the more typical NRF setup in which a pencil photon beam is incident on a sample. The NRF process redirects the pencil beam isotropically so that geometry can be used to distinguish between the incident beam and the fluorescence photons. For the self-irradiating sample case, this will not be the case, as the sample and source are the same.

There are two possible approaches to solving this scenario. In the first approach, one exploits the difference in energy of the incident and fluorescent photons by conducting a transmissionlike measurement. If the isotope of interest is in the sample, then those isotopes will shift the energy of the photons on the resonance to be slightly below the resonance because of the recoil of the nucleus. This process will create a notch in the spectrum exiting the sample that could be exploited by looking for resonant scattering on a secondary target in a manner similar to conducting transmission measurements.

In the second approach, known geometrical differences between the sample and the matrix of material surrounding the sample are exploited. As an example, consider a sphere of ${ }^{235} \mathrm{U}$ surrounded by lead. By determining the difference between spectra with a collimated detector focused on the lead and focused on the ${ }^{235} \mathrm{U}$ sample, one may be able to extract information on the isotopic content of the sample. This approach assumes that the source has gamma rays with energies significantly above the resonant energy. In the case of ${ }^{235} \mathrm{U}$, it might be the $9 / 2$ - state at $46.2 \mathrm{keV}$ that could serve as the resonant state excited by the downscattering of lines from the $186-\mathrm{keV}$ level.

Two scenarios are envisioned for this application. First, consider the ${ }^{235} \mathrm{U}$ sphere inside a $\mathrm{Pb}$ shell. Second, consider a solid sphere of material, composed mostly with iron but mixed with significant quantities of fission products and ${ }^{235} \mathrm{U}$. For both cases, we will assume these measurements are conducted in the laboratory without any significant time constraints (up to a day may be taken to determine whether or not a particular isotope is present). 
The technical challenges are

- Developing technique to exploit NRF signature

- Does the rescattering of the initial gamma rays produce an adequate spectra that can be used for NRF processes

\section{- Gas Samples}

The sampling and analysis of air can provide significant information about activities producing significant gas or aerosol outputs. The current approach frequently involves processing the sample, e.g., chemical separation, to prepare the sample for measurements. Is it possible the NRF may provide a faster method to assay the sample for isotopic content? One advantage of NRF over passive measurements is the identification of stable isotopes as well.

Two scenarios are envisioned: a sample of compressed air inside a container and an aerosol sample collected on filter paper. The measurement will be conducted in the laboratory with measurement time restricted to roughly one day. The geometries will be compact as possible.

Technical challenges are

- Sensitivity to small quantities in a larger matrix

- Sensitivity to small samples

\section{- Tagging}

There are multiple applications in which it is necessary to tag an item to maintain a clear chain of custody. The tagging approach fails if the tag can be easily counterfeited. Is it possible to use NRF techniques to read a tag that would be difficult to counterfeit?

The scenario is one in which tags are used at a nuclear materials storage depot. Containers of SNM are sealed and tagged using an NRF-based tag so that the containers can be tracked. Measurements of the tags will be conducted at the facility with a dedicated device. Measurements will need to be conducted relatively quickly, on the time scale of one minute per tag, as there may be a number of tags that need to be evaluated.

One possible approach for this application is to use transmission measurements to verify the tags. The tag may contain many isotopes which will generate a plethora of NRF peaks. Some of the isotopes may be chosen so that peaks overlap in order to complicate interpretation. By conducting NRF transmission measurements, it may be possible to isolate a handful of isotopes within the sample. It may be possible for the NRF response of those isotopes to be unobservable in scattering NRF measurements due to either background contributions or overlapping resonances within the detector response that hide the resonances.

The technical challenges are

- Measuring small quantities in a larger matrix

- Characterization of suspect material

When a questionable sample of material is initially collected, the first step to studying the sample is to conduct an initial survey of it to gain some knowledge of the contents of the 
sample. This process can be complicated, because the sample may contain multiple layers of material and exactly what materials are present may be completely unknown. It is possible that NRF may be used as a screening tool to conduct the initial survey of the sample to gain some understanding of its contents in a non-intrusive manner.

The scenario is a laboratory measurement conducted on a sample. There is little time constraint, so the measurements must be completed on the time scale of one day. Two examples of possible samples have been taken from Moody [2005]; these are both actual samples on which forensics studies were conducted. The first example is a sample of highly enriched uranium (HEU) powder that was contained in a glass ampoule, which was wrapped in paper, surrounded by wax and encased in a lead pig. The HEU consisted of $72.7 \%{ }^{235} \mathrm{U}$, $13.9 \%{ }^{238} \mathrm{U}, 11.9 \%{ }^{236} \mathrm{U}$ and $1.1 \%{ }^{234} \mathrm{U}$. The second example is a bottle of thorium-nitrate, containing roughly $4 \%$ uranium to thorium. Both samples contained trace amounts of other isotopes orders of magnitude smaller in size than the actinide material itself. If one has direct access to this material, it is likely that one can conduct more sensitive tests of these minor constituents using other techniques. However, it is worthwhile exploring exactly how small a sample NRF can observe within the sample.

The technical challenges

- Sensitivity to small quantities of isotopes in a larger matrix

- Sensitivity through layers of materials

- Three-dimensional isotopic mapping

- Lack of knowledge of NRF signatures for isotopes

\section{- Verification of SNM in a cargo container}

One of the initial motivations for studying the potential of NRF was the potential for detection of nuclear weapon material in intermodal cargo containers. The relatively large geometry of the cargo container, the unknown matrix of materials surrounding the material, the need to conduct the measurements quickly and the low tolerance for false-positive alarms combine to make this application challenging. In this case, we will assume that another process such as photofission or radiography has been used to identify a suspicious region within the cargo container so that one need to only verify that the region contains SNM.

The scenario envisioned is at a fixed facility at a domestic port of entry. The measurement needs to be completed on the time scale of ten minutes. A physical region of interest inside the container has already been identified, so that the measurements may focus on that region. Geometries suitable for the inspection of a cargo container will be assumed. Three different types of material matrices surrounding the SNM will be considered: an empty container to provide a baseline sensitivity, a container filled with $1.4 \mathrm{~g} / \mathrm{cm}^{3}$ Celotex as an approximation for shielding from incidental material, and finally an engineered shield consisting of a moderate amount of lead and borated-polyethylene. The engineered shielding would be adequate to eliminate $95 \%$ of neutrons from plutonium and $95 \%$ of the $1001-\mathrm{keV}$ gamma rays of ${ }^{238} \mathrm{U}$. Both ${ }^{235} \mathrm{U}$ and ${ }^{239} \mathrm{Pu}$ materials will be considered.

The technical challenges are

- Sensitivity to small quantities of isotopes in a larger matrix

- Sensitivity through layers of materials 


\subsection{Research Issues}

One objective of this project is to develop a guide as to the research that needs to be conducted in order for NRF to become a viable tool in addressing national security applications. Sorting through the list of potential applications, we observe that there are only a relatively small number of research issues that are common to multiple applications. In addition to those explicitly listed, there are also issues involving the photon source and the detector. By examining and addressing these issues, one has the potential to impact the viability of NRF as a tool for national security across multiple applications. These issues will be discussed below.

\subsection{Source development}

The photon source is a critical aspect of NRF measurements. In selecting a source, one must balance competing issues such as the source intensity required to achieve necessary count rate, sensitivity to background issues, and complexity of the system. For instance, a laboratory measurement may sacrifice count rate and simplicity of the system in order to increase sensitivity, while a field measurement may sacrifice sensitivity to utilize a simple system.

There are three broad avenues of future source development research:

1. Narrow-bandwidth accelerators promise increased sensitivity due to lower backgrounds and lower dose. Existing systems, which rely on Compton backscattering, are sophisticated machines bound to use in a laboratory. Design of narrow-bandwidth sources that provide suitable intensity and are operationally robust is critical for the long term advancement of NRF as a national security tool.

2. Novel accelerators may provide either a more compact/transportable form factor or a more favorable photon distribution than bremsstrahlung.

3. Evolutionary development of bremsstrahlung sources to provide a higher fraction of the photons on the resonance.

While it is beyond the scope of the project to delve deeply into source issues, the project will specify the source requirements that are required for NRF-based applications to be successful.

\subsection{Increase detector rate}

The instantaneous detector rates on HPGe detectors are a significant limitation for NRF applications. Frequently, measurements are limited by detector rate rather than source intensity, increasing required measurement times. Improvements in throughput of the detector without sacrificing resolution and signal-to-noise would significantly enhance the NRF capabilities.

There are three distinct avenues for improving detector rate. First, one can optimize the filter configuration between the detector and target in an attempt to more effectively filter the lower energy photons. As the dominant rate contributions are at lower energies, this improved filtering would enable increased source intensity. The second approach is to develop a better understanding of the performance of existing commercial products under high rate conditions. PNNL has started this effort by studying the performance of three digital signal analysis packages under high rate conditions. The third avenue is to develop novel high-rate, high-resolution detectors, which, while achieving order of magnitude improvements in rate, also renders it most likely that the detector will require segmentation of some kind. Segmentation may present its own challenge in terms of energy resolution, given the difficulty of maintaining calibrations across many elements. 


\subsection{Increase detector resolution}

For the measurements in which the NRF signature of ${ }^{235} U$ was first observed and quantified, the signal-to-noise of the measurement was roughly 1:1. Given that the resonances are typically $1 \mathrm{eV}$ wide after Doppler broadening, the observed width of the peak in the measurements is determined by the detector resolution, not the NRF response. For HPGe, the resolution is on the order of a $\mathrm{keV}$. Thus, one is losing a factor of 1000:1 in signal-to-noise due to the limitations of current instrumentation. Improved resolution could dramatically improve the sensitivity of NRF measurements.

Signal-to-noise is not the only consideration, however. If the driving consideration were only energy resolution, one could use micro-calorimetry techniques to improve. These systems, however, by definition consist of small amounts of detector material. This small mass greatly reduces detection efficiency, particularly at the higher energy photons involved in NRF measurements. Thus, the ultimate goal is an optimization of both detector efficiency and energy resolution in order to improve performance for NRF measurements.

\subsection{Sensitivity to small quantities of material}

The minimum sample size on which one can currently conduct NRF measurements is on the order of 1 gram. Can research facilities be designed to enable measurements on significantly reduced sample size? For example, a 1-mg ${ }^{235} \mathrm{U}$ sample exposed to a $1 \mathrm{~mA}$ bremsstrahlung photon beam will generate on the order of $100 \mathrm{NRF}$ interactions per second. Allowing for $1 \%$ total detection efficiency, one can in principle obtain a 3\% measurement on the time scale of 20 minutes. Thus, we argue that it is the experimental facility that is limiting the sample size, not the physics. It is conceivable that through proper shielding of the source and the detectors one can conduct measurements on substantially smaller sample sizes.

\subsection{Sensitivity to small quantities of isotopes within a larger matrix}

This issue is similar to sensitivity to small quantities of materials, except that the matrix of materials surrounding the isotopes of interest generates additional continuum background. Are there experimental techniques to improve sensitivity to the isotopes with signals in a large background? For instance, could one conduct coincidence measurements to reduce the background? There are many observed NRF-related excitations that emit multiple gamma-rays as the excited nucleus decays back down to ground state. By designing a system to exploit these coincident signals, one may be able to reduce the minimum detectable quantity of an isotope inside a larger sample. Another approach might be to apply transmission detection or some novel measurement technique to maximize the sensitivity.

\subsection{Signature search}

There are several isotopes for which we do not currently know the NRF signatures. Isotopes of interest include the longer-lived plutonium isotopes, with the exception of ${ }^{239} \mathrm{Pu},{ }^{241} \mathrm{Am}$, and

${ }^{237} \mathrm{~Np}$. University of California Berkeley, in collaboration with PNNL and LLNL, is currently conducting measurements on ${ }^{237} \mathrm{~Np}$.

Two other isotopes of interest are ${ }^{6} \mathrm{Li}$ and ${ }^{9} \mathrm{Be}$. While the nuclear energy levels are known to a limited extent, it is not clear that they will have an NRF signature. In the case of ${ }^{6} \mathrm{Li}$, there is a 
3562-keV state that has been reported to have been observed through $\left(\gamma, \gamma^{\prime}\right)$ measurements [Rasmussen, 1969], yet the internal transition ratio for this level is listed as unknown in NUDAT [2009]. We anticipate that the state is there and can be observed through NRF, but it would be worthwhile repeating these measurements to confirm.

In the case of ${ }^{9} \mathrm{Be}$, there are multiple levels of suitable width but all emit predominantly neutrons. Besides exploiting the small fraction of internal transition probability (e.g., $10^{-6} \%$ for the $3049-\mathrm{keV}$ level), it may be possible to observe the presence of ${ }^{9} \mathrm{Be}$ indirectly. The $3049-\mathrm{keV}$ level of ${ }^{9} \mathrm{Be}$ is $282 \mathrm{keV}$ wide. As the beam passes through the ${ }^{9} \mathrm{Be}$, the $(\gamma, \mathrm{n})$ process will deplete the incident gamma flux near that resonance. By conducting a transmission measurement with the secondary target consisting of materials with one resonance inside and one outside of the $3049-\mathrm{keV}$ resonance of ${ }^{9} \mathrm{Be}$, it may be possible to infer the presence of ${ }^{9} \mathrm{Be}$ on the basis of the difference in scattering from the two resonances in the secondary target. ${ }^{13} \mathrm{C}$ would make an idea secondary target, with one resonance at $3089-\mathrm{keV}$ and one at $3684-\mathrm{keV}$. Experimental tests of this concept may be worth conducting.

\subsection{Three-dimensional Isotopic mapping}

Some applications require the determination of the three-dimensional placement of isotopes. The precision required varies by application. The simplest approach is to raster the beam across the target and use the collimation and placement of the detector and the cone of the beam to define a voxel over which the detector will observe NRF interactions. It may be worthwhile to study how small one can define the voxel. There will be a tradeoff between voxel size and counting rate unless the photon source intensity can be increased to counter the reduction in count rate due to the collimation. Another possible solution to this issue is the development of a CT-type NRF system. By conducting transmission NRF measurements from various angles to the sample, one should be able to assemble a three-dimensional map of the isotopic content of a sample, much like a radiography CT system. This approach may provide the isotopic and geometry information required by the application.

\subsection{Sensitivity through layers of materials}

At times there are samples that consist of multiple layers of materials. For instance, the smuggled HEU sample described earlier was inside a glass ampoule, surrounded by paper, surrounding by wax, placed inside a lead pig. How well does NRF work going through multiple layers of differing materials? How well does NRF work when there are multiple layers of the same material?

\subsection{Sensitivity through large quantities of materials}

One issue frequently encountered in the use of any interrogating source is the attenuation of the source by the material. If the source is significantly attenuated, there may not be enough flux deeper into the material to generate the desired signal. This occurs by design when one shields a radioactive source to protect personnel. As a function of incident photon intensity, what is the amount of material that can be assayed using NRF techniques? Are there sophisticated approaches that could extend the depth that can be probed? At what point is the attenuation of the sample so intense that one cannot conduct a CT NRF measurement? 


\subsection{Conclusion}

This document is intended as a progress report, to outline some of the research questions the project generates as it evaluates the utility of NRF in addressing a variety of national security applications. The next effort is to determine how best to answer the research issues, followed by a prioritization of those questions to ensure that the most important are addressed. These issues will be addressed through either analytical calculations, computer simulations, analysis of previous data or collection of new measurements. It will also be beneficial to conduct a thorough examination of a couple of the more promising applications in order to develop concrete examples of how NRF may be applied in specific situations. The end result will be the development of an understanding of whether the application of NRF is limited by technology or physics in addressing national security applications, to gain a motivation to explore those possible applications, and to develop a research roadmap so that those possibilities may be made reality. 


\subsection{References}

[Bohle, 1984] D. Bohle, "New magnetic dipole excitation mode studied in the heavy deformed nucleus ${ }^{156} \mathrm{Gd}$ by inelastic electron scattering," Phys. Lett. B137, 27 (1984).

[Bertozzi, 2005] W. Bertozzi, R.J. Ledoux, "Nuclear resonance fluorescence imaging in nonintrusive cargo inspection," Nucl. Inst. Meth. B241, 820 (2005).

[Roadmap, 2007] Special Nuclear Materials Movement Detection Portfolio Technology

Roadmap, NA22-PDP-02-2007.

[Moody, 2005] K.J. Moody, I.D. Hutcheon, P.M. Grant, "Nuclear Forensics Analysis," Taylor \& Francis, New York (2005).

[NUDAT, 2009] NuDAT 2.5, http://www.nndc.bnl.gov/nudat2/, National Nuclear Data Center, Brookhaven National Laboratory, Upton, NY (2009).

[Quitter, 2008] Brian Quitter, private communication, University of California, Berkeley (2008).

[Rasmussen, 1969] V.K. Rasmussen and C.P. Swann, "Gamma-Ray Widths in 13C, 6Li and 31P," Phys. Rev. 183, 918 (1969).

[Smith, 2008] L.E. Smith, et al.,"AFCI Safeguards Enhancement Study: Technology Development Roadmap," PNNL-18099 (2008).

[Snyder, 1954] T. M. Snyder and R. W. Williams, "Upper Limit for the Time between Fission and the Emission of Neutrons," Phys. Rev. 81, 171 (1951).

[XCOM, 2009] XCOM, http://physics.nist.gov/xcom. National Institute of Standards and Technology, Gaithersburg, MD (2009).

[Varlamov, 1987] V.V. Varlamov, N.G. Efmikin, V.V. Surgutanov, A.A. Khoronenko, A.P. Chernyaev, "Photofission of Uranium U-235,238", Photonuclear Data (1987).

[Vandenbosch, 1973] R. Vandenbosch, J.R. Huizenga, "Nuclear Fission," Academic Press, New York (1973). 